\title{
Organic electronics: the nuclear option
}

\author{
Dane R McCamey \\ NPG Asia Materials (2015) 7, e156; doi:10.1038/am.2014.117; published online 16 January 2015
}

The conductivity of electronic materials is fundamentally determined by the interaction of its charge carriers with their environment. In devices based on conducting polymers, the dipole-mediated hyperfine interactions of polarons with the ubiquitous hydrogen nuclear spins are thought to be responsible for a range of phenomena including the extremely large magnetoresistive effects that are often seen. ${ }^{1}$ Malissa et al. ${ }^{2}$ have now demonstrated an elegant approach to investigating these effects in working devices by utilizing electron-nuclear double resonance and electron spin-echo envelope modulation, both detected by monitoring the change in the current through a poly[2-methoxy5-(2-ethylhexyloxy)-1,4-phenylenevinylene] (MEHPPV)-based organic light-emitting diode (see Figure 1).

Although conventional magnetic resonance spectroscopy is well suited to investigating electronnuclear interactions in bulk materials, major difficulties exist when applying these techniques to thin-film devices under operating conditions (that is, biased and at room temperature). The number of spins in these devices is small, making detection difficult; conventional device architectures often distort the microwave pulses required for magnetic resonance and it can be challenging to distinguish between active spins, which influence device operation, and passive spins, which are present but have no practical influence.

The approach of the authors-the use of electrical readout in devices designed for the purposeovercomes these issues by allowing a sensitive detection method, which implicitly measures only those spins that impact conductivity. It also allows the interactions to be investigated under device-operating conditions-biased and at room temperature. The effect is confirmed by deuterating one of the polymer side chains, with the expected change in observed resonance frequency.

Challenges remain. First, the short lifetime of the polarons $\left(T_{1} \approx 40 \mu \mathrm{s}\right)$ limits their ability to probe coherent nuclear spin effects, an important step if these material are to be used for storing quantum information (as the authors suggest). Next, the

School of Physics, UNSW Australia, Sydney, New South Wales, Australia

E-mail: dane.mccamey@unsw.edu.au

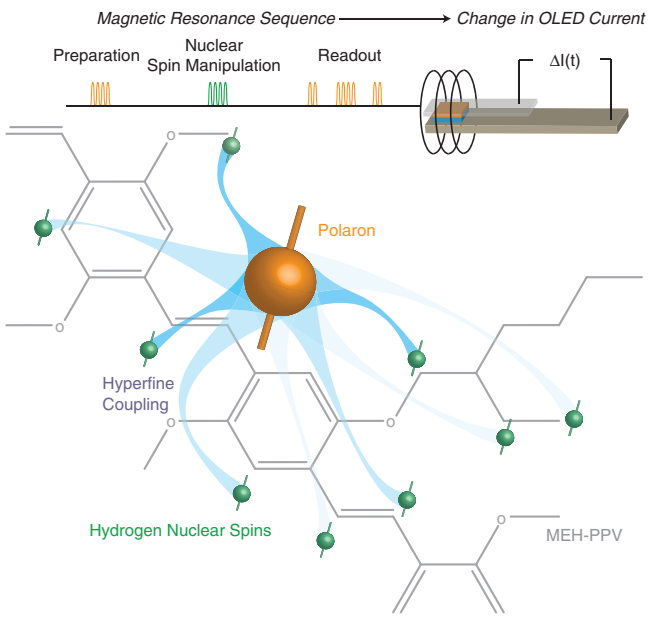

Figure 1 The current through an organic light emitting diode (OLED) is modified following nuclear magnetic resonance on the polymer's hydrogen atoms. A modified electron-nuclear double resonance sequence mediates this coupling. Owing to the long lifetime of nuclear spins, the authors propose that this may provide a way to store and readout information, although significant challenges remain, not least the short electron spin coherence and lifetimes. (Note that not all nucei are shown here.)

approach is best suited to systems with coupling strengths that give resolved hyperfine resonances, ${ }^{3}$ but the material used here does not satisfy this condition due to the separation between the polaron (whose wavefunction resides mainly on the backbone) and the nuclei (which are mostly on the side chains). Nevertheless, the approach demonstrated here provides a useful technique for quantifying electron-nuclear interactions in a wide range of organic devices and materials and should find widespread application.

1 Mermer, Ö., Veeraraghavan, G., Francis, T., Sheng, Y., Nguyen, D., Wohlgenannt, M., Köhler, A., Al-Suti, M. \& Khan, M. Large magnetoresistance in nonmagnetic $\pi$-conjugated semiconductor thin film devices. Phys. Rev. B 72, 205202 (2005)

2 Malissa, H., Kavand, M., Waters, D. P. van Schooten, K. J., Burn, P. L., Vardeny, Z. V., Saam, B., Lupton, J. M. \& Boehme, C. Room-temperature coupling between electrical current and nuclear spins in OLEDs. Science 345 , 1487-1490 (2014).

3 McCamey, D. R., van Tol, J., Morley, G. W. \& Boehme, C. Electronic spin storage in an electrically readable nuclear spin memory with a lifetime $>100$ seconds. Science 330, 1652-1656 (2010).

(c) (i) (2) This work is licensed under a Creative Commons Attribution-NonCommercial-ShareAlike 4.0 International License. The images or other third party material in this article are included in the article's Creative Commons license, unless indicated otherwise in the credit line; if the material is not included under the Creative Commons license, users will need to obtain permission from the license holder to reproduce the material. To view a copy of this license, visit http:// creativecommons.org/licenses/by-nc-sa/4.0/ 